\section{Computer system for recording and display of data from newborn infants with respiratory illnesses}

Computers have been extensively used to process data from adults with serious illnesses such as coronary heart disease (Farr et al., 1972; Hulberman et al., 1972; Lewis et al., 1972). Few reports of the use of computers in neonatal intensive care units have appeared (Clark et al., 1971; Perlstein et al., 1976). We have used a computer to record and display data from newborn infants with respiratory illnesses. The system has been designed to (1) collect brief clinical details; (2) collect values for arterial bloodgas tensions, acid-base status, and variables relating to continuous positive airway pressure (CPAP) and mechanical ventilation; and (3) display the respiratory data in graphical form both on television screens in the neonatal intensive care unit and on paper, for permanent storage in the case notes. We report here our experience with this system during a period of 25 months.

\section{Methods}

Computer. The computer is a PDP $11 / 45$ machine located in the Medical Physics Department of this hospital. It is connected by cables to a terminal in the neonatal unit. The computer is also used by the Departments of Radiotherapy and Nuclear Medicine. Special equipment has been designed to allow the computer to process data from all three departments simultaneously. Technical details have been published elsewhere (Clifton et al., 1976).

The terminal in the neonatal unit, which consists of two television screens (Fig.) and a typewriter keyboard, is sited in the centre of the unit, adjacent to the blood-gas analyser.

Data-handling. When an infant is admitted to the unit the terminal is activated by pressing a key. A succession of questions then appears on one of the screens, inquiring about the infant's case number, sex, gestational age, birthweight, and date and time of birth. If the infant has been transferred from another hospital, data are entered describing events during the journey. All the questions are answered by a simple keying procedure, for example a ' $Y$ ' means yes and a ' $N$ ' no. Numerical values such as birthweight are typed directly on to the keyboard. The data are then displayed for checking.

When these data have been entered, a list of options appears on the screen. If the option for the entry of the case history is selected, a list of the common diagnoses appears and a shorthand way of entering them is available, for example hyaline membrane disease can be keyed as 'A1'. Unusual diagnoses are typed in by name. A similar procedure is used for entering $x$-ray appearance, and whether or not the infant is being given total parenteral nutrition, has a pneumothorax or a coagulation defect, is infected, or is being treated with antibiotics.

Once the basic clinical information has been supplied, the main use of the computer is for the recording and display of blood gas and other data from infants with respiratory illnesses. When samples of arterial blood have been analysed, values for inspired $\mathrm{O}_{2}$ concentration $\left(\mathrm{F}_{\mathrm{r}} \mathrm{O}_{2}\right)$, arterial $\mathrm{O}_{2}$ tension $\left(\mathrm{PaO}_{2}\right), \mathrm{CO}_{2}$ tension $\left(\mathrm{PaCO}_{2}\right), \mathrm{pH}$, and base excess are entered, again in response to a series of questions posed by the computer. If the equilibration method is used for calculating $\mathrm{PaCO}_{2}$ and base excess the computer will automatically do the calculation from the Sigaard-Anderson nomogram (Siggard Anderson, 1962).

Information is supplied about whether the infant is breathing spontaneously, or being treated by CPAP or mechanical ventilation. When CPAP is used, the method (endotracheal tube or face mask) and the applied pressure, are entered. If the infant is mechanically ventilated the data include peak airway pressure, end-expiratory pressure, respiratory rate, and either inspiration-expiration ratio, if the infant's ventilation is controlled, or inspiratory time, if intermittent mandatory ventilation (Kirby et al., 1972) is in use. As when details of the infant's case history are entered, the keying procedure is very simple. If any value lies outside the expected range, the computer will draw attention to it, and ask for confirmation that it is correct.

When data concerning the infant's ventilatory status have been supplied they are displayed (Table) and the computer will then plot the data in graphical form on the screens (Fig.). The time axis is adjusted

Table Details of one entry of mechanical ventilator variables, blood gas tensions, and acid-base status, as shown on the television screen

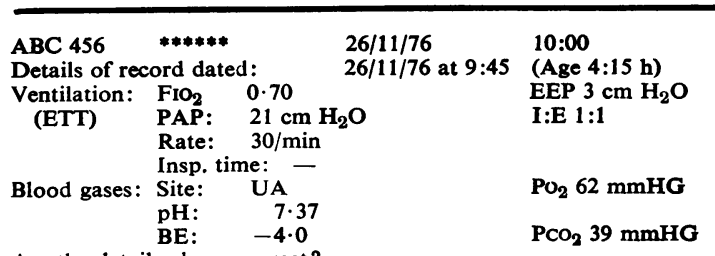

Are the details above correct?

ETT $=$ endotracheal tube; $\mathrm{FIO}_{2}=$ fractional inspired oxygen concentration; $\mathbf{E E P}=$ end-expiratory pressure; $\mathbf{P A P}=$ peak airway pressure; $\mathbf{I}: \mathrm{E}=$ inspiration :expiration ratio; $\mathbf{U A}=$ umbilical artery; $\mathbf{P O}_{2}=$ arterial oxygen tension; $\mathbf{B E}=$ base excess; $\mathbf{P C O}_{2}=$ arterial carbon dioxide tension. 


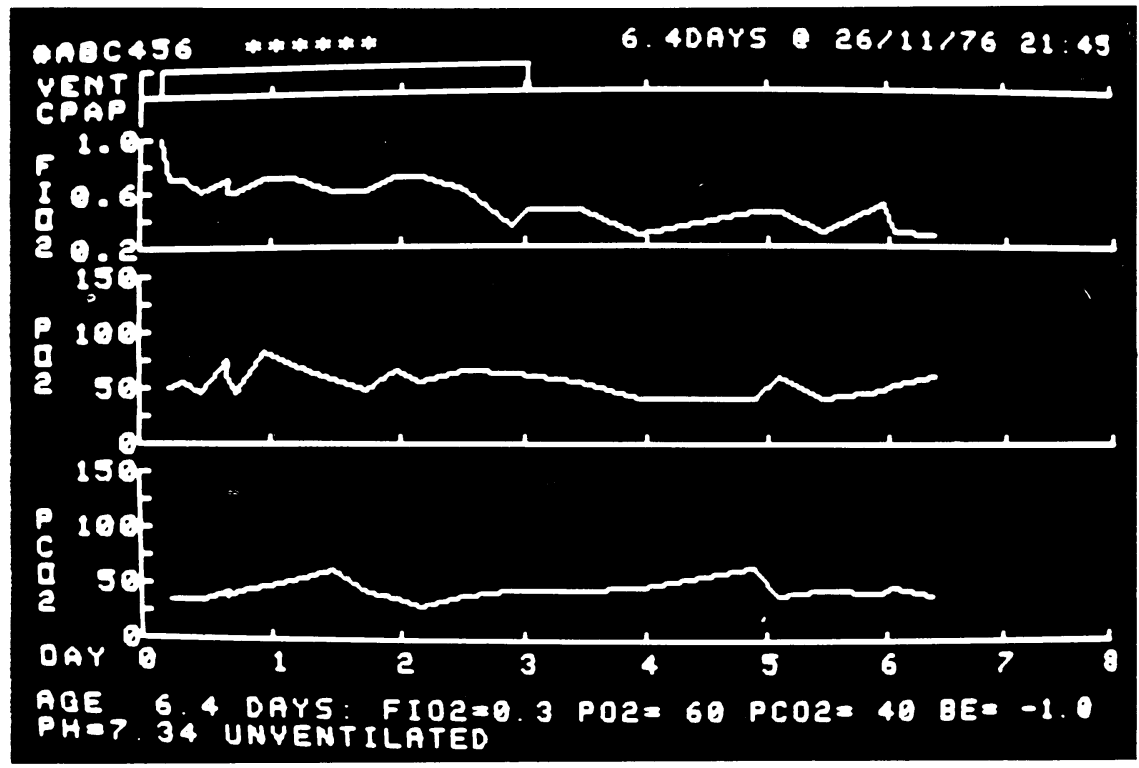

(a)

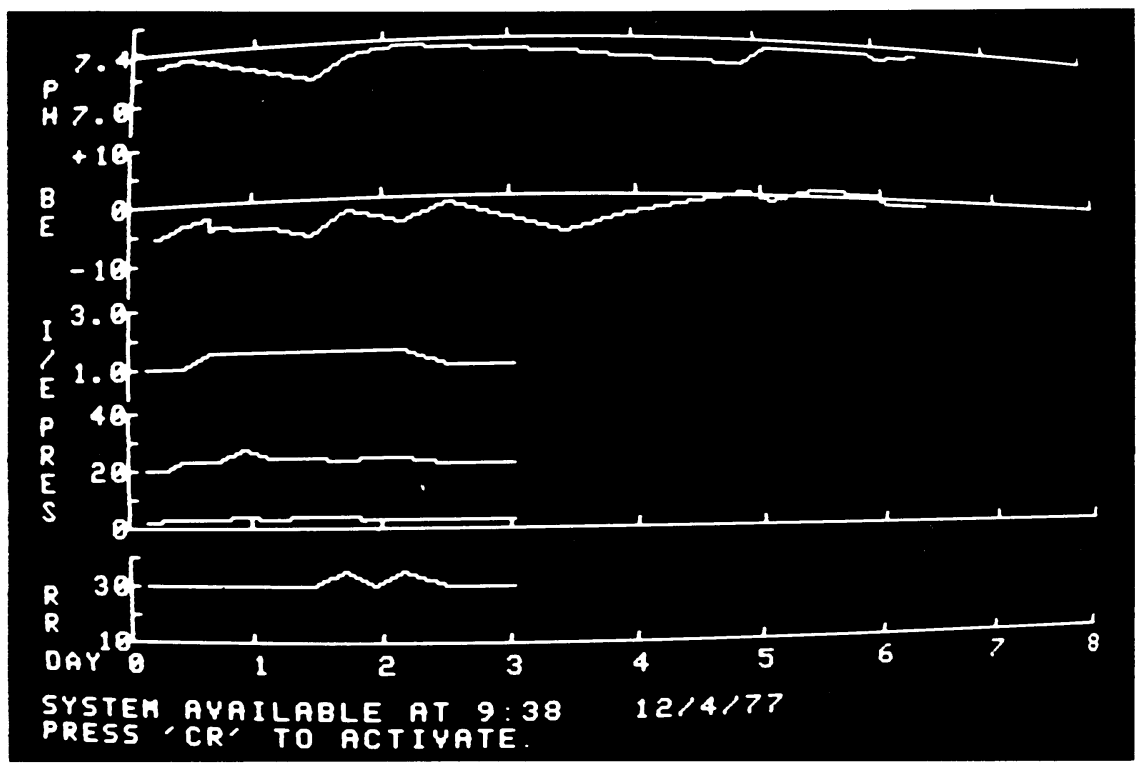

(b)

Fig. The two television screens at the computer termial. The information displaved from above downwards on the upper screen (a) is as follows. The infant's name (deleted here), and age; whether or not mechanical ventilation or

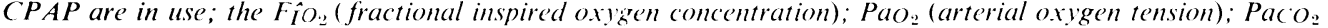
(arterial carbon dioxide tension); and the most recent numerical data. On the lower screen (b) the data are $\mathrm{pH}$; $B E$ (base excess); and then variables relating to mechanical ventilation-I:E (inspiration:expiration ratio); peak airway pressure; end-expiratory pressure; and respiratory rate. The data are displayed on a common time-axis, in this instarce 8 days. 
automatically up to a maximum of $\mathbf{3 0}$ days, but it can, if required, easily be altered by hand. The most recent data are retained in numerical form on the upper screen (Fig.). Lists of data including all the numerical values for blood gases and CPAP or mechanical ventilator variables can also be displayed at will, and any errors can be removed.

The terminal will accept for display data from 6 'active' infants at a time. If data from more than this number need to be entered, one or more infant's data can be temporarily removed from the display at the terminal and stored in the computer 'library' for later recall and revision.

When an infant is discharged or dies, final diagnoses or post-mortem findings are supplied. The data are then permanently stored in the library. Graphs of data are printed on paper at the computer site in the Medical Physics Department and filed in the case notes. Information about infants whose data are in the library can be recalled and displayed at the terminal by typing the number of the case notes on the keyboard. If the computer is not on-line for any reason, patient files cannot be revised, and data from infants in the library cannot be recalled at the terminal, but data from the 6 'active' infants are always available for inspection.

\section{Results}

Infants studied. During the 25-month period, November 1974 to December 1976, data from 277 infants - all those admitted with significant respiratory illnesses-were entered into the computer, usually by the medical staff of the neonatal unit. The most frequent diagnosis was hyaline membrane disease.

Evaluation of use of computer terminal. Analysis done automatically by the computer over a period of 19 months showed that it was on-line and available for the entry of data for $78 \%$ of the total time. The remaining time was used mainly for further developments of the system. During this 19-month period data from 187 babies were collected. A total of 4310 sets of blood gas and related data were entered, giving an average of 23 sets for each infant.

Ten consecutively admitted infants were selected for an evaluation of time saving. After performing the blood gas analysis, two of us (both clinicians) timed, using a stop-watch, how long it took to plot the data by hand for the patient's case notes, and how long it took to enter the same data into the computer and for graphs to be generated on the television screens at the terminal. The entry of 159 sets of data was timed in this way and it was found that the mean time taken to process the data by hand was $2 \cdot 99 \pm \mathrm{SD} 0 \cdot 80$ minutes, versus $1 \cdot 29 \pm 0 \cdot 42$ minutes, using the computer. This difference is significant $(P<0.001)$ and shows a $44 \%$ saving of time when using the computer.

\section{Discussion}

During the 25-month period that the computer terminal has been in routine use in the neonatal unit it has improved the efficiency of the unit. For example, trends in the progression of respiratory illnesses could easily be seen at all times, and medical and nursing staff starting a new shift could be rapidly brought up to date by inspecting the data displayed at the terminal. The clinical staff of the unit were almost entirely responsible for entering the data, thus demonstrating that a computer facility of this type can be managed without difficulty by clinicians. The staff were spared the necessity for writing all the blood gas values and related information in each infant's case notes and constructing graphs by hand. Time saving was clearly shown.

The terminal has proved valuable for undergraduate and postgraduate teaching, since the ways in which the respiratory illnesses of infants in the unit were being treated could be summarised very simply from data on the screens. Since similar data from all 277 infants admitted during the 25-month period under review were available for recall at the terminal, the respiratory management of unusual cases-such as hydrops fetalis-could also be shown if required.

The computer has also proved useful for assessing the performance of the unit and for clinical research purposes. For example, when CPAP and mechanical ventilation by face mask had been in use for 9 months it was possible to obtain from the computer a list of treated infants, mean values for the variables that we wished to analyse, and the outcome for the infants (Allen et al., 1975). More recently, the computer has been used in the analysis of data from a controlled trial of early intervention with CPAP (Allen et al., 1977). To analyse these data by hand would have been extremely time consuming.

Since the computer system has functioned well, and gained acceptance in the unit, we propose to enlarge its capability by using it to process additional variables, such as continuously recorded arterial oxygen tension (Conway et al., 1976), transcutaneous. oxygen tension (Jolly et al., 1976), and blood pressure.

We conclude that a computer terminal for the display and analysis of data from sick infants in a neonatal intensive care unit improves the efficiency of the unit, is valuable for teaching and research, and should be made available to other units that admit large numbers of seriously ill infants, such as regional: referral centres. 


\section{Summary}

A computer system has been used for the recording and display of data from 277 infants with respiratory illnesses consecutively admitted to a neonatal intensive care unit during a period of 25 months. Improved efficiency of data handling was demonstrated, and the system proved valuable for teaching and research.

\section{References}

Allen, L. P., Blake, A. M., Durbin, G. M., Ingram, D., Reynolds, E. O. R., and Wimberley, P. D. (1975). Continuous positive airway pressure and mechanical ventilation by facemask in newborn infants. British Medical Journal, 4, 137-139.

Allen, L. P., Reynolds, E. O. R., Rivers, R. P. A., le Souëf, P. N., and Wimberley, P. D. (1977). Controlled trial of continuous positive airway pressure given by face-mask for hyaline membrane disease. Archives of Disease in Childhood, 52, 373-378.

Clark, J. S., Veasy, L. G., Jung, A. L., and Jenkins, J. L. (1971). Automated $\mathrm{PO}_{2}, \mathrm{PCO}_{2}$ and $\mathrm{pH}$ monitoring of infants. Computers and Biomedical Research, 4, 262-274.

Clifton, J. S., Ingram, D., Todd-Pokropek, A. E., and Allen, L. P. (1976). Special purpose computer systems for intensive care applications. Real-time Computing in Patient Management. Ed. by D. W. Hill and J. P. Payne. Peregrinus, London.

Conway, M., Durbin, G. M., Ingram, D., McIntosh, N., Parker, D., Reynolds, E. O. R., and Soutter, L. P. (1976). Continuous monitoring of arterial oxygen tension using a catheter-tip polarographic electrode in infants. Pediatrics, 57, 244-250.

Farr, F. L., Clark, J. S., Gardner, R. M., and Veasy, L. G. (1972). Using a dedicated small computer in conjunction with a time-shared system in a hospital intensive care unit. Computer and Biomedical Research, 5, 535-539.

Hulberman, M., Kamm, B., Tate, N., and Osborn, J. J. (1972). An evaluating computer-based patient monitoring system at Pacific Medical Center. Computers and Biomedical Research, 5, 535.

Iolly, P., Reynolds, E. O. R., and Soutter, L. P. (1976). Comparison of transcutaneous oxygen tension $\left(\mathrm{tcPO}_{2}\right)$ and arterial oxygen tension $\left(\mathrm{PaO}_{2}\right)$ in newborn infants. (Abst.) Pediatric Research, 10, 890.

Kirby, R., Robison, E., Schultz, J., and deLemos, R. (1972). Continuous flow ventilation as an alternative to assisted or controlled ventilation in infants. Anesthesia and Analgesia, 51, 871-875.

Lewis, J. F., Deller, D., Quinn, M., Lee, B., Will, R., and Raines, J. (1972). Continuous patient monitoring with a small digital computer. Computers and Biomedical Research, 5, 411-428.

Perlstein, P. H., Edwards, N. K., Atherton, H. D., and Sutherland, J. M. (1976). Computer-assisted newborn intensive care. Pediatrics, 57, 494-501.

Sigaard Anderson, O. (1962). The pH-log $\mathrm{PCO}_{2}$ blood acidbase nomogram revised. Scandinavian Journal of Clinical and Laboratory Investigation, 14, 598-604.

L. P. Allen, J. S. Clifton, D. Ingram, P. N. le SOÜ̈F, E. O. R. ReYNolds, and P. D. WimberLeY Departments of Paediatrics, and Medical Physics and Bioengineering, University College Hospital, London.

Correspondence to Professor E. O. R. Reynolds.

\section{Effect of phototherapy on thermal environment of the newborn}

The aim of this study was to investigate the effects of the radiant energy emitted by phototherapy lights on the thermalenvironment of incubator- and cot-nursed babies, and to determine whether there were situations in which babies might be exposed to adverse thermal conditions.

\section{Materials and method}

The 40 babies included in this study were undergoing phototherapy for hyperbilirubinaemia in the neonatal unit of the City Hospital, Nottingham. Incubator-nursed babies had a mean birthweight of $1.94 \mathrm{~kg}( \pm 0.15 \mathrm{SEM})$ and were studied at an average age of 4 days. Cot-nursed babies were heavier (mean birthweight $3.01 \pm 0.12 \mathrm{~kg}$ ) and were studied at an average age of 6 days. The jaundice was considered to be physiological in all cases, but aggravated by prematurity in 13. In a further 3 babies bruising or cephalhaematoma was present. Infants with Rhesus or ABO incompatibility were not included.

Phototherapy was administered with either the Vickers unit, containing four $58 \mathrm{~cm} 40$ watt tubes, or the Air Shields unit, containing eight $58 \mathrm{~cm} 20$ watt tubes. Both units were used either $60 \mathrm{~cm}$ above the mattress of an open cot, or $40 \mathrm{~cm}$ above the mattress of an incubator.

Ambient temperatures were measured with thermocouples and recorded by a Cambridge multichannel recorder. The room temperature, mattress surface temperature, and air temperature $2 \mathrm{~cm}$ above the mattress were measured continuously. The suitability of the operative environmental temperature was assessed by measuring the baby's rate of oxygen consumption. An open circuit oxygen consumption apparatus has been developed that does not require moving the baby to a metabolic chamber (Smales, 1978).

All measurements were made $1 \frac{1}{2}$ hours after a feed. Incubator air temperature was recorded from the thermometer in the canopy of the incubator and an allowance made for radiant heat loss according to the temperature of the room air (Hey and Mount, 1967). In 10 babies, nursed naked in incubators, the rate of $\mathrm{O}_{2}$ consumption was measured after an hour's phototherapy. After the following feed phototherapy was discontinued and a second measurement of $\mathrm{O}_{2}$ consumption was made an hour later. Incubator temperature varied by less than $0.8^{\circ} \mathrm{C}$ for each pair of measurements.

In another 20 babies the rate of $\mathrm{O}_{2}$ consumption was measured after an hour's phototherapy over an 